\title{
Neuronal Sensitivity to Microsecond Time Disparities in the Electrosensory System of Gymnarchus niloticus
}

\author{
Atsuko Matsushita and Masashi Kawasaki \\ Department of Biology, University of Virginia, Charlottesville, Virginia 22904
}

To perform the jamming avoidance response (JAR), the weakly electric fish Gymnarchus detects time disparities on the order of microseconds between electrosensory signals received by electroreceptors in different parts of the body surface. This paper describes timedisparity thresholds of output neurons of the electrosensory lateral line lobe (ELL), where the representation of timing information is converted from a time code to a firing-rate code. We recorded extracellular single-unit responses from pyramidal cells in the ELL to sinusoidally modulated time disparity with various depths $(0-200 \mu \mathrm{s})$. Threshold sensitivity to time disparities measured in 123 units ranged from 0.5 to $100 \mu \mathrm{s}$ and was $\leq 5 \mu \mathrm{s}$ in $60 \%$ of the units. The units from pyramidal cells in the inner and outer cell layers of the ELL responded equally well to small time disparities. The neuronal thresholds to time disparities found in the ELL are comparable with those demonstrated in behavioral performance of the JAR. The sensitivity of ELL units to small time disparities was unaffected when the center of the cyclic time-disparity modulation was shifted over a wide range (up to $250 \mu \mathrm{s}$ ), indicating an adaptation mechanism for steady-state time disparities that preserves the sensitivity to small dynamic changes in time disparities. Phase-locked input neurons, which provide time information to the ELL by phase-locked firing of action potentials, did not adapt to steady-state time shifts of sensory signals. This suggests that the adaptation emerges within the ELL.

Key words: electric fish; binaural comparison; coincidence detection; electrosensory lateral line lobe; jamming avoidance response; temporal coding

\section{Introduction}

Many animal species are known to exhibit behaviors that require a sensory capability of detecting small time disparities ${ }^{a}$ on the order of $10^{-8}$ to $10^{-5} \mathrm{~s}$ between sensory inputs (Knudsen et al., 1979; Mason et al., 2001; Simmons et al., 2003) (for review, see Carr and Friedman, 1999; Carr et al., 2001). Sensory organs in these animals inform the CNS of the moment of sensory events by the occurrence times of action potentials in the afferent nerve [for temporal coding, see Theunissen and Miller (1995)]. The behaviorally important time disparities in the afferent action potentials are then detected by central neurons that compare the times between inputs. These time comparator neurons inform higher centers of the brain of the time disparity by their firing rate (impulse-rate coding).

The weakly electric fish Gymnarchus and Eigenmannia generate quasi-sinusoidal electric organ discharges (EODs) from their tail at a few hundred hertz. When the fish's EODs are jammed by EODs from a neighboring fish, the fish shifts its discharge fre-

Received Aug. 30, 2005; revised 0ct. 29, 2005; accepted 0ct. 30, 2005

This work was sponsored by grants to M.K. from the National Science Foundation (IBN-0235533) and Precursory Research for Embryonic Science and Technology, Japan Science and Technology Corporation.

"The terms "time" and "phase" will be used interchangeably in this paper following a convention in the electric fish literature. Although phase, $\varphi$, should formally be expressed by the unit of degrees, it will be often expressed in time, $t$. $\left(\varphi / 360^{\circ}=f \times t\right.$, where $f$ is the frequency of the carrier signal.) "Time disparity" and "phase difference," for example, are synonymous.

Correspondence should be addressed to Masashi Kawasaki, Department of Biology, University of Virginia, Gilmer Hall, P.0. Box 400328, Charlottesville, VA 22904-4328. E-mail: mk3u@virginia.edu. DOI:10.1523/JNEUROSCI.3670-05.2005

Copyright $\odot 2005$ Society for Neuroscience $\quad$ 0270-6474/05/2511424-09\$15.00/0 quency away from that of the neighbor to avoid jamming of its electrolocation system (Watanabe and Takeda, 1963; Bullock et al., 1972, 1975). This jamming avoidance response (JAR) requires time disparity between sensory feedback signals of its own EODs that are differentially time modulated (or phase modulated) at different skin areas by the neighbor's EODs (Bastian and Heiligenberg, 1980; Heiligenberg and Bastian, 1980; Kawasaki, 1993). The time-disparity threshold for correct performance of the JAR is $10^{-7}$ to $10^{-6} \mathrm{~s}$ (Rose and Heiligenberg, 1985; Guo and Kawasaki, 1997). The occurrence times of feedback sensory signals from the EODs are sampled by a type of electroreceptor in the skin that sends phase-locked action potentials to the electrosensory lateral line lobe (ELL) in the brain via S-type afferents (hereafter simply S-afferents) in Gymnarchus (Guo and Kawasaki, 1997). The time disparities between the S-afferents are detected by a neuronal circuit in the ELL (Fig. 1) (Kawasaki and Guo, 1996; Matsushita and Kawasaki, 2004), in which the output of pyramidal cells modulate their firing rates in response to time disparities between S-afferents from different skin regions.

A behavioral study in Eigenmannia suggested that the microsecond time sensitivity of the JAR requires spatial summation of sensory signals (Rose and Heiligenberg, 1985) and neurons showing comparable time sensitivity were found only in the last stage (diencephalon) of the sensory hierarchy (Kawasaki et al., 1988; Rose et al., 1988). Kawasaki and Guo (1996), however, reported that neurons in an early stage of time processing (hindbrain) may exhibit microsecond sensitivity in Gymnarchus. In the present study, we examined the threshold sensitivity of pyramidal 


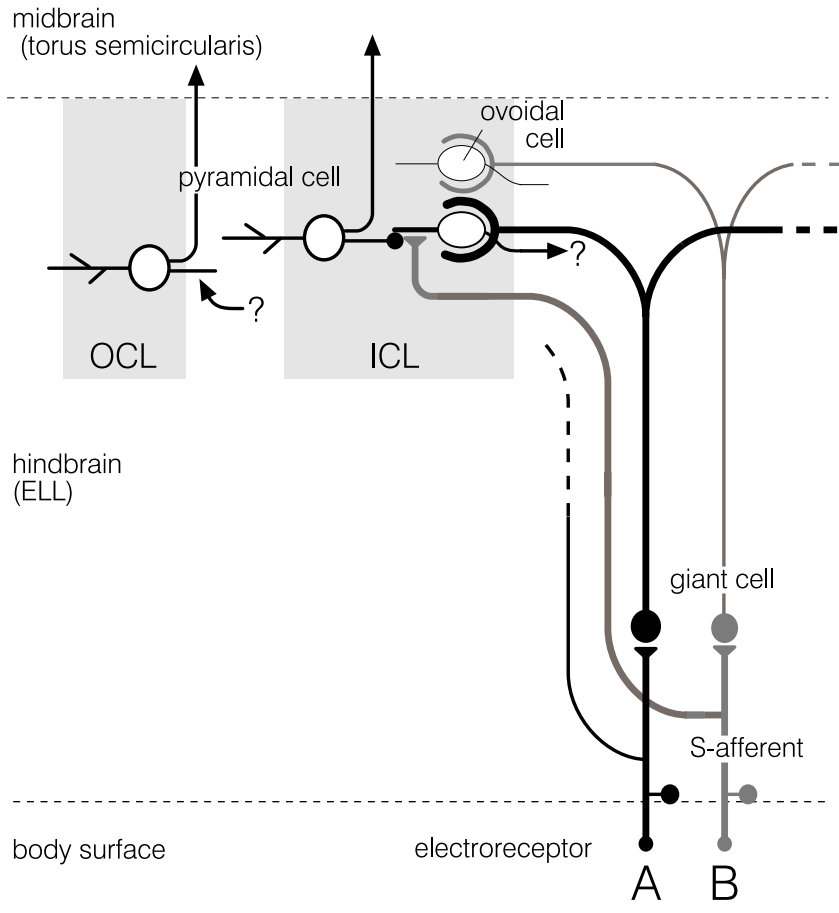

Figure 1. Neural circuit in the ELL for computation of differential phase modulation (time disparity) between different body regions $A$ (black line) and B (gray line). The time information of $A$ and $B$ is presented to the ICL directly by $S$-afferents and indirectly via giant cells. In the ICL, an ovoidal cell receives these information, and pyramidal cells also receive inputs from S-afferents. The pyramidal cell in the ICL changes its firing rate in response to the differential phase modulation. The pyramidal cell in the $0 \mathrm{CL}$ also responds to differential phase modulation. The projection sites of the axons of ovoidal cells are unknown. Sources of inputs to the pyramidal cells in OCL are also unknown. Modified from Matsushita and Kawasaki (2004).

cells in the ELL of Gymnarchus using a "phase chamber" in which stimuli presented to different skin areas were precisely controlled in time.

\section{Materials and Methods}

Animals. Approximately 50 Gymnarchus niloticus (total length, 12-21 $\mathrm{cm}$ ) were used. Environmental conditions in the holding tanks were identical to those described previously (Kawasaki, 1994). After anesthesia with tricaine methanesulfonate (MS-222; 1:10000; Sigma, St. Louis, $\mathrm{MO}$ ), we immobilized fish with an intramuscular injection of flaxedil (gallamine triethiodide; $8-15 \mu \mathrm{l}$ of a $0.3 \mathrm{mg} / \mathrm{ml}$ solution; Sigma), which greatly attenuated EOD amplitude. The silenced EOD was replaced with an artificial electrosensory stimulus as described below. Activity of the EOD pacemaker command signal was recorded from the tail to monitor the fish's condition throughout experiments. Fish were placed inside a plastic chamber (phase chamber; $12 \times 18 \times 5 \mathrm{~cm}$ ) set in a plastic tank $(50 \times 50 \times 13 \mathrm{~cm})$, gently held with a sponge-lined clamp, and submerged in water, except for a small area along the surface of the head. Water resistivity was set between 5 and $8 \mathrm{k} \Omega \cdot \mathrm{cm}$. Oxygen-saturated water perfused the gills via a mouthpiece inserted in the mouth. After local application of $2 \%$ lidocaine, a small hole was opened in the skull above the hindbrain, exposing the dorsal surface of the ELL (see below). These procedures were approved by the Institutional Animal Care and Use Committee at the University of Virginia.

Electrosensory stimulation in the phase chamber. We applied electrosensory stimuli using a phase chamber, which electrically isolates the head and trunk into separate compartments using a high-resistance partition (Fig. 2A). This partition was placed immediately behind the pectoral fin and was sealed against the fish's body using petroleum jelly. In the head compartment, a sinusoidal carrier signal that mimicked the silenced EOD was given between an electrode placed in the mouth and a pair of carbon rod electrodes straddling the head. In the trunk compartment, a
A

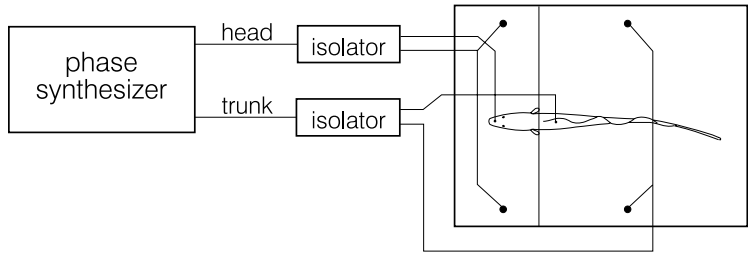

B

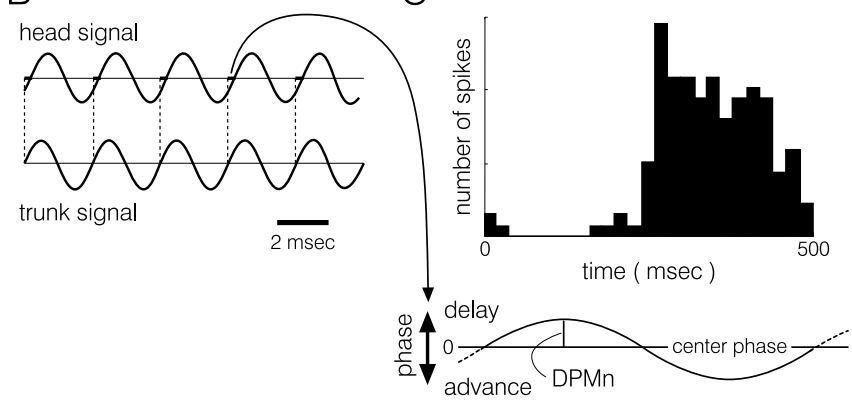

Figure 2. $\quad A$, Electrosensory stimulation in the phase chamber. The phase synthesizer generated two sinusoidal signals (shown in $\boldsymbol{B}$ ) at an identical frequency that stimulated the head and trunk compartments. The signals were applied between an internal electrode and two electrodes straddling the fish in each compartment. $\boldsymbol{B}$, Phase synthesizer generated small phase shifts between the head and trunk signal. The phase shift of the head signal was sinusoidally modulated as detailed in C. C, An example of a spike histogram constructed relative to the $2 \mathrm{~Hz}$ phase modulation cycle. Because this unit fired more to phase advance and less to phase delay, the unit was called a head advance-preferred unit. The sinusoidal modulation of phase was typically centered at zero (as shown) but could be shifted by up to $\sim 180^{\circ}$. DPMn depicts the depth of differential phase modulation at $n \mu$ s.

sinusoidal stimulus with an identical frequency was given between a pin electrode inserted into the dorsal musculature near the anterior end of the dorsal fin and a pair of carbon rod electrodes straddling the trunk. The signals were delivered to each compartment via a homemade isolator with field effect transistors and adjusted to a stimulus amplitude of 1-2 $\mathrm{mV} / \mathrm{cm}$ measured near the skin surfaces. Signal cross-talk between the two compartments was less than $-30 \mathrm{~dB}$, as measured outside the fish's body.

A phase synthesizer (Wavetek 650) generated an unmodulated sinusoidal signal for the trunk and a phase-modulated sinusoidal signal for the head, creating differential phase modulation (DPM) between the two compartments (Fig. $2 \mathrm{~B}, \mathrm{C}$ ). DPM was sinusoidal such that the phase of the head was delayed during the first half of the modulation cycle $(0-$ $180^{\circ}$ ) and advanced for the second half of the cycle $\left(180-360^{\circ}\right)$. The phase modulation was cyclic and continuous, and its frequency was typically $2 \mathrm{~Hz}$. DPM was controlled by an analog sinusoidal signal that was generated by a digital-to-analog converter (DA3-4; Tucker-Davis Technology, Gainesville, FL) and applied to the differential phase modulation port of the phase synthesizer. The center of the sinusoidal phase modulation (center phase) was typically zero but could be changed $\pm 180^{\circ}$. Various depths of DPM were used. DPM at a depth of $n \mu$ s will be expressed as DPMn (e.g., DPM5 for DPM with a modulation depth of 5 $\mu \mathrm{s})$. Various depths between DPM0 and DPM200 were applied in a random sequence to determine the response threshold for each unit. The threshold sensitivity of a unit to DPM was defined by the minimum depth of DPM with which the unit showed a significant response (see below, Data acquisition and analysis). DPM50 at $2 \mathrm{~Hz}$ was used as a search stimulus.

The accuracy and stability of the electrosensory stimulus generated by the phase synthesizer were measured by a time interval counter with absolute time accuracy of 1 ns (SR620; Stanford Research Systems, Sunnyvale, CA). The head and trunk sinusoidal signals generated by the phase synthesizer were directly fed into Schmitt trigger inputs of the time interval counter, and zero-crossing times of the signals were recorded over $10^{4}$ cycles. The SD of period jitter of the unmodulated head signal 
A
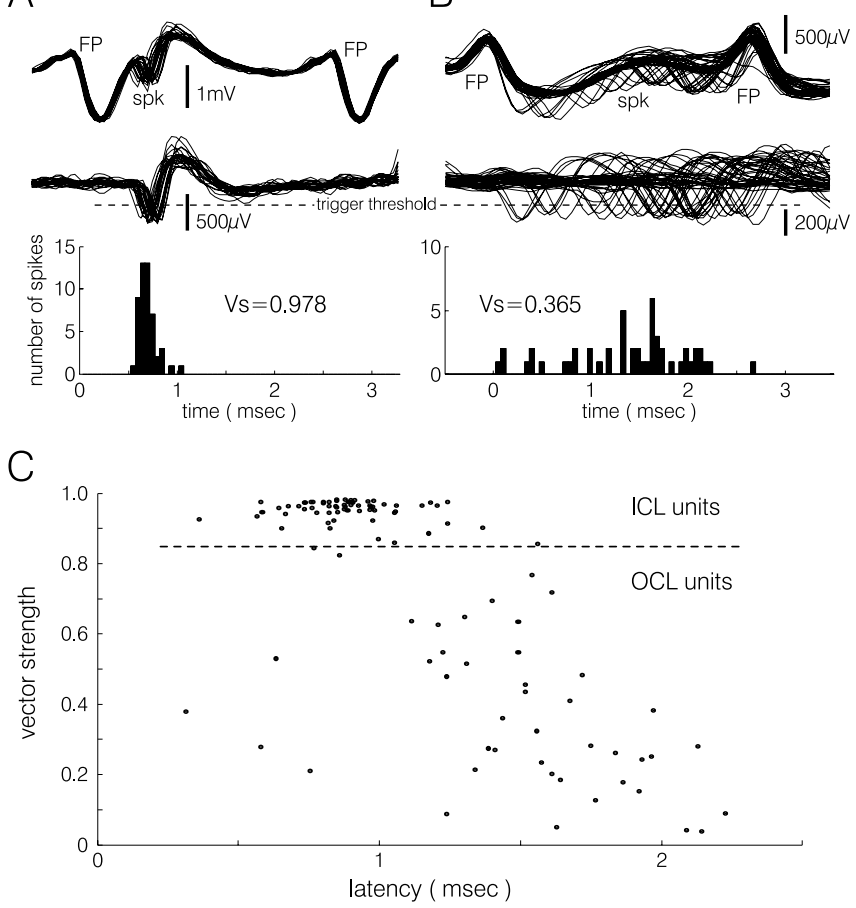

Figure 3. Isolation of spiking activities from the field potential and vector strength. $\boldsymbol{A}$, Raw signal from the recording electrode (top trace, 25 traces are superimposed) includes periodic field potential (FP) that was phase locked to electrosensory sinusoidal stimulation and spikes (spk) from an ICL unit. Spikes were isolated by a delay-cancellation technique (second trace). Spike histogram (bottom) shows the degree of synchronization (Vs) of spiking to the carrier signal. $\boldsymbol{B}$, A similar example from an 0 CL unit (50 traces are superimposed). $\boldsymbol{C}$, Relation between V and response latency of spikes measured from the negative maximum slope of the field potential $(n=108)$. The dashed line at $\boldsymbol{V} \boldsymbol{s}=0.84$ separates the units into the pyramidal cell in the ICL or OCL, based on a previous study.

was 178 ns. The SD of phase difference between the two signals at DPM0 was 288 ns. The smallest phase modulation that could be achieved by the analog input of the phase synthesizer via its analogto-digital converter was $81 \mathrm{~ns}$.

Electrophysiological recording. Extracellular recording was made with glass capillary electrodes with a carbon fiber (diameter, $10 \mu \mathrm{m}$ ) in most experiments [modified from Fu and Lorden (1996)]. A borosilicate glass capillary with no center filament was pulled on a Brown/Flaming electrode puller (P-97; Sutter Instruments, Novato, CA) after inserting a carbon fiber into the capillary. One side of the separated capillaries retained the carbon fiber that was subsequently cut by a scalpel and extruded by $5-10 \mu \mathrm{m}$ from the glass end. Some units were recorded with glass capillary electrodes filled with $3 \mathrm{M} \mathrm{NaCl}$ (tip diameter, $\sim 8 \mu \mathrm{m}$ ) or with indium-filled and gold/platinum-plated glass capillary electrodes (tip diameter, $\sim 10 \mu \mathrm{m}$ ) (Dowben and Rose, 1953).

Extracellular single-unit activity ( $50 \mu \mathrm{V}$ to $1 \mathrm{mV}$ ) and large field potentials (see below, Recording site) caused by phase-locked firing of primary afferents and giant cells (up to $10 \mathrm{mV}$ ) were amplified $10 \times$ with an amplifier (model 5A; Getting Instruments, Iowa City, IA). The large periodic field potentials (see below) were eliminated by a cancellation technique in which the raw signal was delayed by one period, inverted in sign, and added to the original signal with a digital signal processor (sampling frequency, $48 \mathrm{kHz}$; model DSP-4; QSC Audio Products, Costa Mesa, CA). The resulting signal, which contained only nonperiodic signals, was sent to a Schmitt Trigger circuit, which in turn triggered an event timer (ET1; Tucker-Davis Technologies) that generated time stamps for spiking at $1 \mu$ s resolution (Fig. $3 A, B$ ).

Intracellular recordings from primary electrosensory phase-locking neurons ( $\mathrm{S}$-afferents and giant cells) were made in the deep fiber layer (DFL) of the ELL (see below, Recording site) using sharp glass-capillary electrodes $(30-50 \mathrm{M} \Omega$, filled with $3 \mathrm{M} \mathrm{KCl})$. The electrode was lowered into the DFL, from which large field potentials can be recorded as determined by preliminary extracellular recordings (Kawasaki and Guo, 1996). Light vibration of the electrode established the penetration into a single neuron. When stable phase-locked action potentials were obtained, we started a program that lasted $30 \mathrm{~s}$ : the phase in the carrier signal was held at $0 \mu \mathrm{s}$ for the first $5 \mathrm{~s}$, then shifted to either +100 or -100 $\mu \mathrm{s}$ for $20 \mathrm{~s}$, and finally shifted back to $0 \mu \mathrm{s}$ for $5 \mathrm{~s}$ (see Fig. 10, bottom traces). We recorded spike times and analyzed spike phase shifts.

Recording site. The ELL is a hindbrain structure that consists of bilateral lobes, the anterior half and medial edges of which are covered by the valvula cerebelli (Bass and Hopkins, 1982). The ELL consists of three distinctive zones: dorsal, ventral, and medial (MZ), each of which is layered. The DFL constitutes the core of the ELL and is shared by all zones. In the MZ, the DFL is covered with an inner cell layer (ICL), outer fiber layer, outer cell layer (OCL), and molecular layer, in this order from the core. The MZ occupies most of the caudal half of the ELL, and as the zone extends rostrally, the lateral extent of the MZ becomes smaller. We placed electrodes in the posterior half of the MZ, which corresponds to the region between panels $G$ and $H$ of Figure 1 in Kawasaki and Guo (1998). The recording site could be determined by monitoring the waveform of the large field potentials (up to $10 \mathrm{mV}$ ) generated by phaselocking neurons in the DFL (giant cells and S-afferent terminals) [as shown in Kawasaki and Guo (1996), their Fig. 2].

Data acquisition and analysis. Spike histograms were constructed relative to the stimulus modulation period (typically $500 \mathrm{~ms}$ ) (Fig. 2C), and the following response parameters were computed for each histogram: average spike rate $=$ (total number of spikes) $/$ (total duration of stimulation); F1, the Fourier component of the histogram at the modulation frequency; F2, the Fourier component of the histogram at twice the modulation frequency (second harmonic) (F1 and F2 are expressed in the unit of spikes/s); and $\boldsymbol{V a}$, the mean vector angle of spiking relative to the modulation cycle, which was computed as follows:

$$
\boldsymbol{V a}=\arctan \left(\sum_{i=1}^{n} x_{i} \times \sin _{i} / n / \sum_{i=1}^{n} x_{i} \times \cos _{i} / n\right),
$$

where $x_{i}$ is the spike count of the $i$ th bin in a histogram. $\cos _{i}=\cos (i \cdot$ $2 \pi / n), \sin _{i}=\sin (i \cdot 2 \pi / n), i=1, \ldots, n . n$ is the total number of bins in the modulation histograms (Goldberg and Brown, 1969). Va takes a value between 0 and $360^{\circ}$ and indicates "response center" where spikes are most likely to occur in a modulation cycle. $\boldsymbol{V a}=90$ and $270^{\circ}$ correspond to the points with the largest phase delay and advance of the head signal with respect to the trunk signal, respectively. The vector strength of spiking relative to the carrier cycle, Vs, was computed as follows:

$$
\boldsymbol{V s}=\sqrt{\left(\sum_{i=1}^{n} x_{i} \times \cos _{i} / n\right)^{2}+\left(\sum_{i=1}^{n} x_{i} \times \sin _{i} / n\right)^{2}},
$$

where $x_{i}$ is the spike count of the $i$ th bin in a histogram constructed relative to the period of the carrier signal. $i=1, . ., n . n$ is $1 / f \times 10^{6}(f$, frequency of the carrier signal). Vs expresses the degree of synchronization of spikes to the carrier signal and takes a value between 0 and 1 . Values of $\boldsymbol{V} \boldsymbol{s}$ of 0 and 1 indicate, respectively, no and complete synchronization (phase locking) to the carrier signal.

Statistical significance of spike responses to small phase modulation was examined by the $V$ test $(\alpha=0.05)$ and Rayleigh $z$ test $(\alpha=0.05)$. The $V$ test examines whether the observed circular distribution of spike times relative to the phase modulation cycle has a tendency to cluster around a hypothetical angle, which was obtained as the value of $\boldsymbol{V a}$ for a suprathreshold modulation depth for the unit (Batschelet, 1981). The $z$ test examines the tendency of spiking to occur at any particular angle of phase modulation (Batschelet, 1981).

A computer program written in Matlab controlled the experiments. It controlled the digital-to-analog converters for phase and amplitude modulation, collected time stamps of spikes, and displayed a raster plot, histograms, and statistical data on-line. 

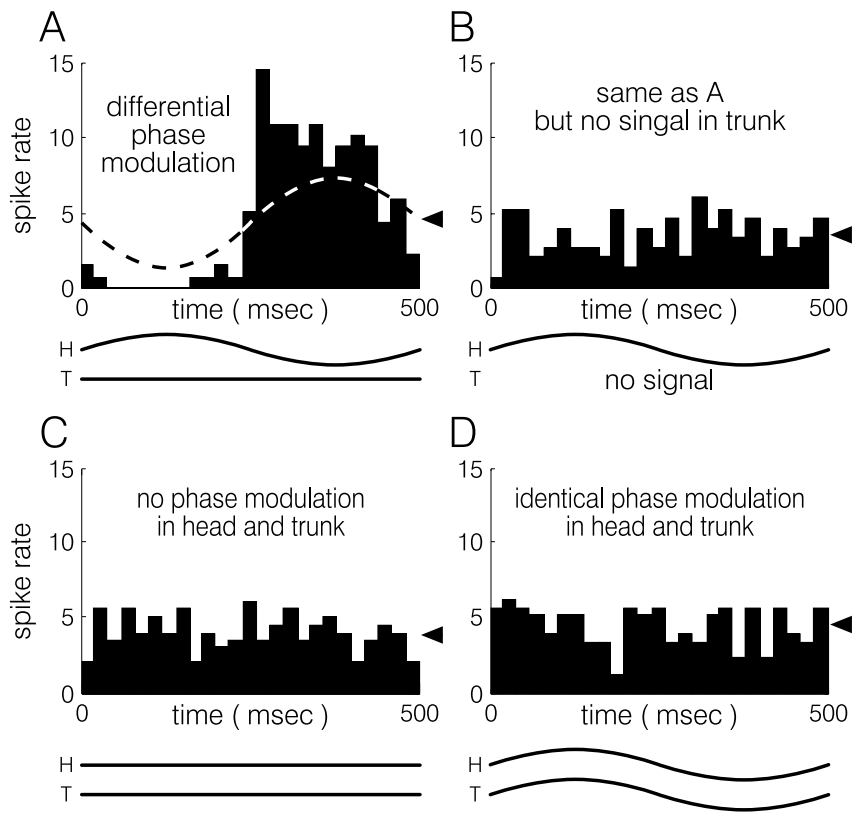

Figure 4. Response of a typical unit recorded in the medial zone of the ELL showing head advance-preferred sensitivity to differential phase modulation. The one cycle sinusoidal lines below each response histogram indicate phase delay (upward deflection) and phase advance (downward deflection) of the head $(\mathrm{H})$ and trunk $(\mathrm{T})$ compartments. The straight lines indicate no phase modulation. $\boldsymbol{A}-\boldsymbol{D}$, An example of an $0 \mathrm{CL}$ unit with $\boldsymbol{V} \mathbf{s}=0.40$. $\boldsymbol{A}$, Differential phase responses to phase advance of $\mathrm{H}$ in reference to $\mathrm{T}$ that had no modulation. $\boldsymbol{B}, \mathrm{H}$ had the same phase modulation as in $\boldsymbol{A}$ but no carrier signal in T. C, Spontaneous discharge with no modulation in $\mathrm{H}$ and T. $\boldsymbol{D}$, Identical phase modulation in $\mathrm{H}$ and $\mathrm{T}$. Note similar mean firing rates in all histograms (arrowheads on the right vertical axes indicate mean firing rate). Depth of phase modulation was $50 \mu$ s in all cases. The broken sinusoidal curve in $\boldsymbol{A}$ is the fundamental Fourier component of the spike histogram. Amplitude and mean of the curve indicate F1 and mean spike rate, respectively.

\section{Results}

Single-unit recordings were made from $\sim 250$ neurons in the medial zone of the ELL. All neurons showed a spontaneous discharge [spike rate, $9.82 \pm 9.10$ (mean \pm SD)] when no differential phase modulation was given. Spike times of these units during spontaneous discharges were analyzed relative to the local field potential that reflects periodic phase-locked firing of the giant cells and afferent fibers (Fig. 3). Mean latency of the spike measured from the time point where the negative slope of the field potential is greatest was strongly clustered around $0.9 \mathrm{~ms}$ for a population of neurons. These units also highly clustered around $V \boldsymbol{s}=0.95$, indicating strong phase locking to the local field potential (Fig. $3 A, C$ ). We regard these units as responses from the pyramidal cells in the ICL based on the previously reported strong correlation between $\boldsymbol{V s}(\boldsymbol{V} \boldsymbol{s}>0.84)$ and morphology/location of the neuron (Kawasaki and Guo, 1998). Other units had longer and more distributed latencies with smaller $\boldsymbol{V s}(\boldsymbol{V} \boldsymbol{s} \leq 0.84)$ (Fig. $3 B, C$ ). These units were regarded as pyramidal cells of the OCL, again based on the distribution of Vs. We call these two populations of units ICL and OCL units, respectively.

\section{Responses to differential phase modulation}

Units recorded in the medial zone of the ELL typically showed responses to sinusoidal modulation of differential phase by modulating their firing rate (e.g., more firing to phase advance and less firing to phase delay) (Fig. $4 A$ ). The units responded neither when a phase-modulated signal was applied to only one compartment (Fig. $4 B$ ), nor when two identical signals were applied to
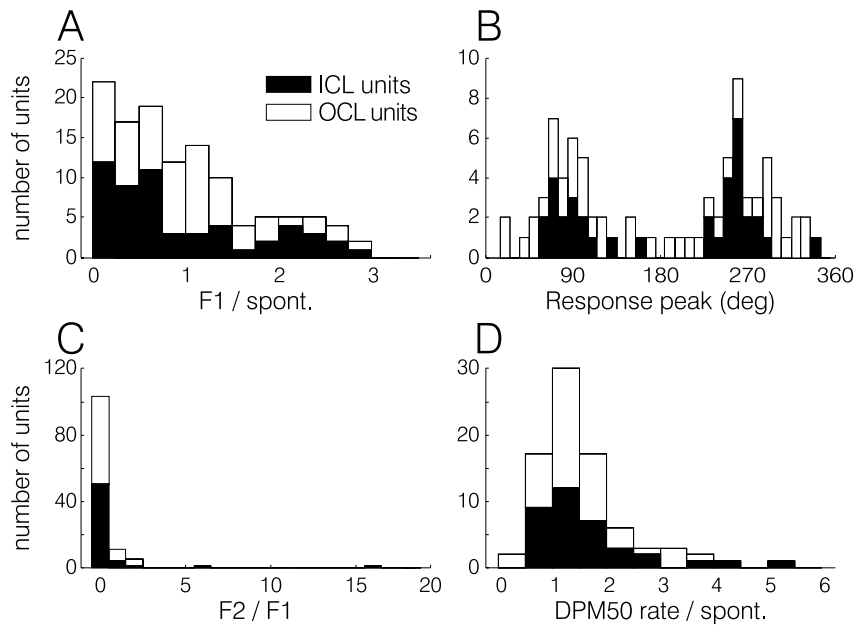

Figure 5. Profiles of ELL units in response to DPM50. $A$, Strength of firing rate to phase modulation in reference to spontaneous discharge rate (F1/spont.). $\boldsymbol{B}$, Response peak in the histogram expressed as angular position of the phase modulation (DPM50) in the units that showed F1/spont $>0.5(n=82)$. C, Only a few neurons showed a histogram peak both at delay and advance parts of the phase modulation yielding large values of F2/F1. D, Comparison of overall rate of spiking during DPM50 and spontaneous discharges. The mean of spike rates of those units with good responses to phase modulation (F1/spont. $>0.5$ ) was $1.63 \pm 1.00$ (minimum, 0.86) times larger than the spontaneous rates. The keys in $\boldsymbol{A}$ apply also to $\boldsymbol{B}-\boldsymbol{D} . \boldsymbol{A}, \boldsymbol{C}$, and $\boldsymbol{D}$ are based on 121 units.

both compartments (Fig. 4C,D), confirming that the units responded to phase differences between the compartments ( $\mathrm{Ka}$ wasaki and Guo, 1996). The fundamental Fourier component of the spike histograms was used as a measure of response strength (Fig. 4A, dashed line). One hundred twenty-one units were tested with DPM50, and the response parameters are summarized in Figure 5. Eighty-two of these ( 36 of 57 from ICL and 46 of 64 from OCL) units responded well (F1/spontaneous discharge rate $>$ 0.5 ) to DPM50 (Fig. 5A). These units showed maximum firing to either phase delay or advance of the head signal in reference to the trunk signal (Fig. 5B). The head delay- and head advancepreferred units occurred with an equal likelihood (ICL units, delay/advance $=20 / 16, p=0.382$; OCL units, delay/advance $=$ $23 / 23, p=0.5$; sign test). Most of the units responded antagonistically to delay and advance, resulting in a weak second harmonic $(\mathrm{F} 2 / \mathrm{F} 1 \approx 0)$ (Fig. $5 C$ ). A small number of the units, however, increased or decreased their firing rate both to phase delay and advance, resulting in a large $\mathrm{F} 2 / \mathrm{F} 1$ ratio (Fig. $5 C$ ). The remaining 39 of the 121 units studied did not respond well to phase modulations even larger than DPM100, and some of them responded well to amplitude modulation. These units probably represent either differential phase-sensitive neurons with their receptive fields not arranged for head-trunk comparisons or amplitudesensitive neurons in the OCL (Kawasaki and Guo, 1998). The mean spike rate to DMP50 was $\sim 1.6$ times higher than spontaneous discharges (Fig. 5D), indicating that the overall responses of the pyramidal cells is excitatory. ICL and OCL units did not show any significant difference in response properties.

\section{Sensitivity to differential phase modulation}

Sensitivity of 123 ELL units was tested with various depths of phase modulation. Figure 6 shows response histograms of six representative units. Distribution of threshold DPMs is shown in Figure 7. Seventy-four units showed statistically significant responses to DPM5 (60\%), 22 units showed statistically significant responses to DPM1 (18\%), and 3 units showed statistically sig- 

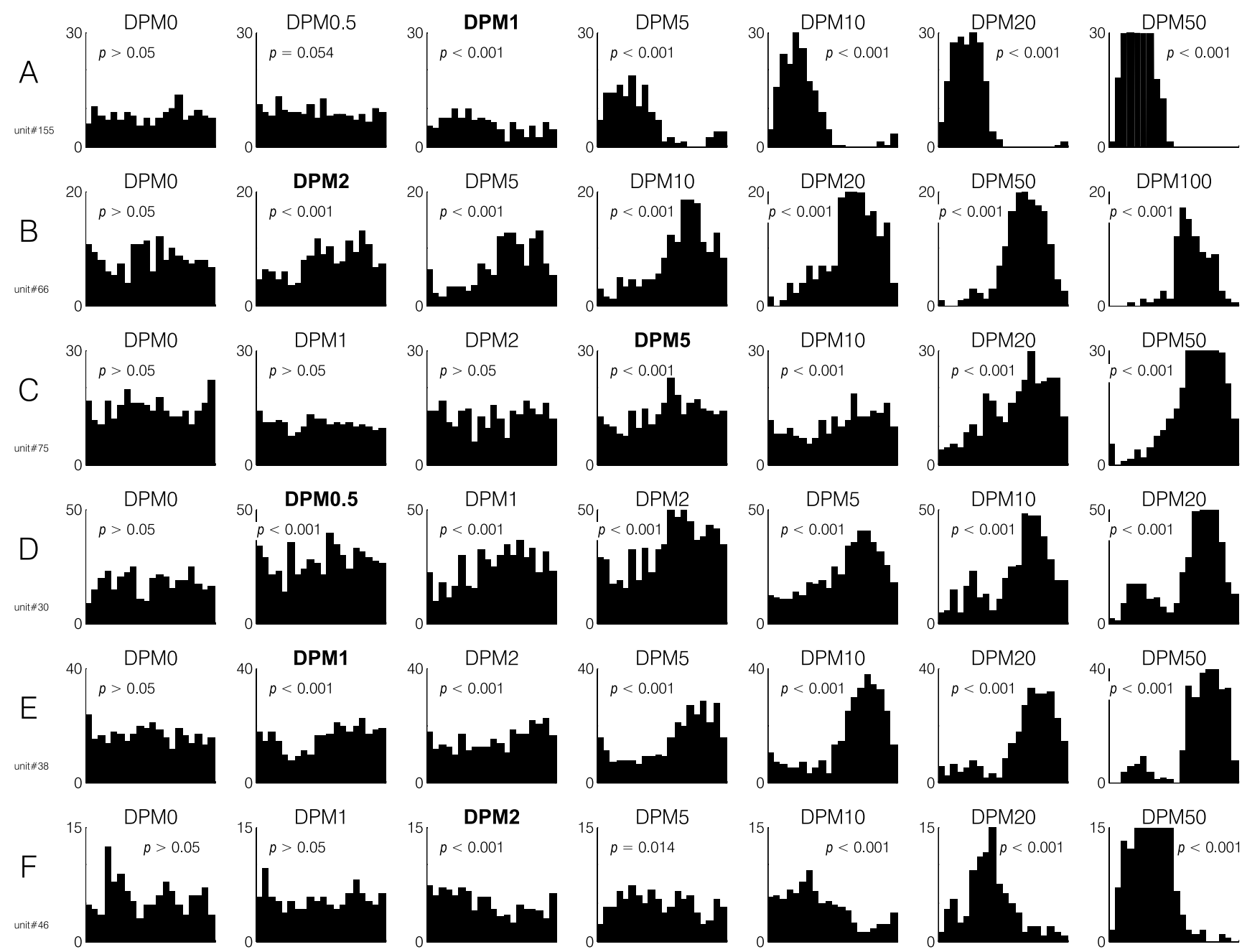

Figure 6. $A-F$, Six representative units examined by various depths of DPM. The abscissas represent one modulation cycle of DPM at $2 \mathrm{~Hz}$ as in the histograms in Figures 2 and 4 . The ordinates show spike rate. $p$ values are from $V$ test. DPMn at the top of each histogram indicates the depth of phase modulation. DPMn in bold letter indicates the threshold of the unit, determined by $V$ test at $\alpha=0.05$. $\boldsymbol{A}-\boldsymbol{C}$, ICL units; $\boldsymbol{D}-\boldsymbol{F}, 0 \mathrm{CL}$ units. $\boldsymbol{A}$ and $\boldsymbol{F}$ are head delay-preferred units; $\boldsymbol{B}-\boldsymbol{E}$ are head advance-preferred units.

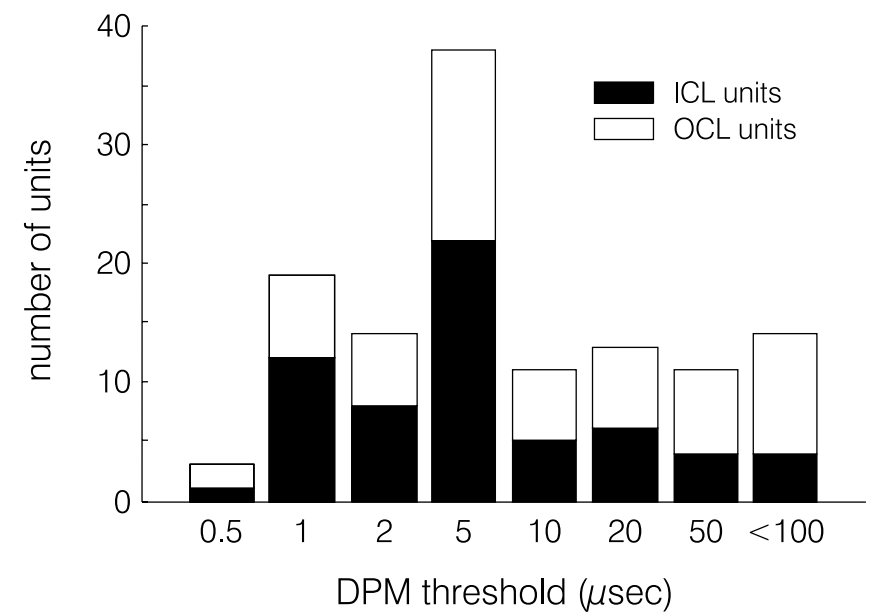

Figure 7. Distribution of thresholds to DPM. ICL and OCL units appear with equal likelihood in each bin (binomial test).

nificant responses to DPM0.5 (2\%). There was no significant difference in thresholds between ICL and OCL units (Fig. 7). These threshold sensitivities are determined by averaged responses to continuously applied sinusoidal phase modulation
( $\sim 60$ cycles). To determine the number of cycles necessary for obtaining a significant response, increasingly larger numbers of cycles were resampled from the same data until a statistically significant response was achieved for each unit (Fig. 8). At DPM5, $24 \%$ of units (18 of 74) showed significant responses within $1 \mathrm{~s}$ (two cycles) (Fig. 8A). At DPM1, 59\% (13 of 22) of units showed significant responses within $10 \mathrm{~s}$ ( 20 cycles) (Fig. $8 B$ ). No difference was noted when the resampling of data were performed with randomly chosen cycles rather than the experimental sequence of cycles. This indicates that no sensitization or desensitization occurred during the sequential presentation of phase modulations.

\section{Adaptation to shifted center phases}

A previous study showed that the differential phase-sensitive neurons in the ELL preserved their sensitivity to suprathreshold phase modulation even after the center value of the sinusoidal phase modulation (Fig. $2 C$, center phase) was shifted by a large amount ( $200 \mu \mathrm{s})$ (Kawasaki and Guo, 1996). In the current study, we examined near-threshold sensitivity of the neurons with shifted center phases. Seventeen units were tested with DPM5 using various center phases (eight ICL units and nine OCL units). After each shifting of the center phase, the units were allowed to adapt for 15-30 s before collecting data. In response to the shifts of center phase, units showed initially either facilitation 
A

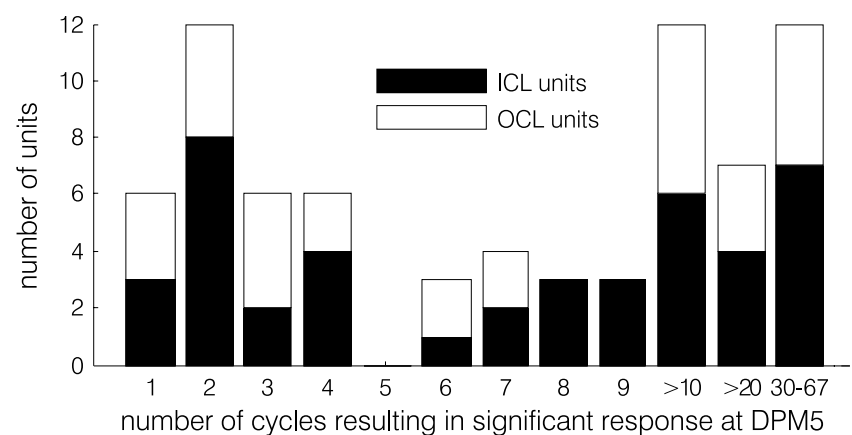

B

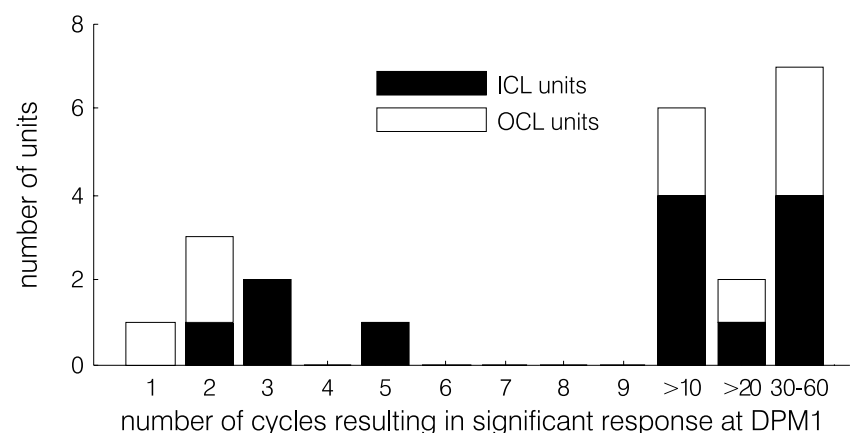

Figure 8. Distribution of the number of cycles needed to yield statistically significant responses to DPM5 (A) and DPM1 (B). ICL and OCL units appear with equal likelihood in each bin (binomial test).

or suppression of firing rate for a few seconds (Kawasaki and Guo, 1996). The responsiveness to DPM5 was preserved over a wide range of center phase. Figure 9 shows representative response histograms at various center phases of three units. The thresholds of these neurons were $\leq 5 \mu \mathrm{s}(p<0.001$ with DPM5, $z$ test) at a center phase of $0 \mu$ s. The neuron shown in Figure $9 A$ remained sensitive to DPM5 with a preference for phase advance for a range between -100 and $+200 \mu$ s of center phases. Sensitivity was lost beyond this range. The response center of this neuron remained constant across all histograms with significant responses $\left(\boldsymbol{V} \boldsymbol{a}=269 \pm 16.4^{\circ} ; n=10\right)$. The neuron shown in Figure $9 B$ remained sensitive to DPM5 with a preference for phase advance between 0 and $200 \mu$ s of center phases $\left(\boldsymbol{V a}=264 \pm 12.1^{\circ}\right.$; $n=7)$. The responsiveness was lost between -20 and $-50 \mu$ s and reappeared at $-100 \mu$ s with a $180^{\circ}$ shifted $\boldsymbol{V a}\left(\boldsymbol{V a}=79.6^{\circ}\right)$. This type of shift in $\boldsymbol{V a}$ was observed in only two units with DPM5 but was more frequently observed with larger DPMs ( $\geq 10 \mu \mathrm{s}$; data not shown). Figure $9 C$ shows a unit that remained sensitive with a similar $\boldsymbol{V a}\left(\boldsymbol{V a}=301 \pm 27.0^{\circ} ; n=12\right)$ over an exceptionally wide range of center phases $(-200$ to $400 \mu \mathrm{s})$. On average, the range of center phases within which units kept the sensitivity to DPM5 was approximately $\pm 125 \mu \mathrm{s}$. As shown in Figure $9 B^{\prime}$, the peak of $\mathrm{F} 1$ was often slightly displaced from $0 \mu$ s, indicating that maximum response strength is not always at a center phase of zero. The displacement of this maximum was usually within \pm $100 \mu \mathrm{s}(n=10$, both ICL and OCL). When the center phase was shifted in a step without sinusoidal phase modulation, units initially showed facilitation or suppression similarly to cases with sinusoidal phase modulations presented above. After a few seconds, the discharge returned to the level of the spontaneous before the step change. The responses then reappeared when tested with small phase modulation. We conclude that sinusoidal phase modulation is not necessary for the adaptation.

\section{Phase-locking neurons do not adapt to steady phase shift}

The results above indicate that pyramidal cells in both ICL and OCL are insensitive to steady changes of the center phase and preserve the sensitivity to dynamic changes of small phase differences. Does this adaptation take place in the phase-locking neurons? The firing times of phase-locking neurons may adapt back to the original phase after a steady phase shift is introduced. To determine whether this adaptation occurs within the ELL, we recorded from phase-locking neurons (S-afferent fibers and giant cell axons) (Kawasaki and Guo, 1996; Matsushita and Kawasaki, 2004) while applying steady phase shifts in the electrosensory carrier signal to one compartment of the phase chamber. Figure 10 shows representative plots of the spike times of a phaselocking neuron in response to steady phase shifts. After an initial $5 \mathrm{~s}$, the phase of the stimulus signal in the head compartment was shifted by either +100 or $-100 \mu$ s and held for $20 s$ and then shifted back to the original phase $(0 \mu \mathrm{s})$. The spike times shifted in response to the shift in phase of the sensory signal remained shifted and showed no adaptation during the $20 \mathrm{~s}$ period. The amount of shift in spike times varied from neuron to neuron. This is probably because, in some neurons, a portion of the receptive field was exposed to the signal in the other chamber, which had no phase shift. When the phase of the carrier signal was shifted in the opposite direction, the spike times of the neuron showed an equally strong shift in the opposite direction (Fig. 10).

\section{Discussion}

\section{Threshold of time disparity}

The major findings of the current study are (1) $60 \%$ of recorded pyramidal cells in the ELL of Gymnarchus are sensitive to $5 \mu \mathrm{s}$ of time disparity between sensory signals applied to the head and trunk, and (2) the sensitivity to sinusoidally modulated time disparity is preserved when a much larger steady-state time disparity is introduced between the head and trunk.

The time disparity between phase-locking neurons that provide time information to the time comparator in the ELL is expected to be smaller than the time disparity between the sensory signals applied to the head and trunk compartment. This is because giant cells, a type of phase-locking neurons, possess a large receptive field that spans across the head and trunk compartments, causing them to fire at an intermediate phase between the head and trunk signal (Matsushita and Kawasaki, 2004). The phase-locking neuron presented in Figure 10 exemplifies such a phase shift that is smaller than that of the stimulus. Therefore, the measured sensitivities of the ELL neurons are conservative. Behavioral and neuronal recording studies (Kawasaki, 1993; Kawasaki and Guo, 2002) demonstrate that the JAR occurs with phase differences between any body surfaces (head-trunk, leftright, etc.), and different ELL neurons are tuned to detect phase differences with different spatial orientations. In the current study, however, we examined time-disparity sensitivity of all encountered pyramidal cells exclusively with the head-trunk configuration alone. We assume that most pyramidal cells would respond to $5 \mu$ s of time disparity if the best orientation of the neurons for phase difference were used (Kawasaki and Guo, 2002). Rose and Heiligenberg (1986) found a comparable level of time-disparity sensitivity in midbrain neurons of the gymnotiform electric fish Eigenmannia, which has evolved its electrosensory system and JAR independently of the mormyriform Gymnarchus (Lauder and Liem, 1983). They showed that some of the 
midbrain neurons were sensitive to time disparities of $\sim 10 \mu$ s using a relatively insensitive statistical method.

Rose and Heiligenberg (1985) discovered that the JAR occurs with time disparities at $10^{-7}$ to $10^{-6} \mathrm{~s}$ in Eigenmannia and argued that integration of sensory information from all body surfaces was required for this sensitivity. The current study in Gymnarchus demonstrates, however, that the individual pyramidal cells in the ELL, which are only one or two synapses away from the phase-locking neurons and receive no substantial spatial integration, are sensitive to $5 \mu \mathrm{s}$. Neuronal integration in higher brain levels (midbrain and diencephalon) to which the pyramidal cells project (Kawasaki and Guo, 1998) may result in even higher sensitivity, as in Eigenmannia (Kawasaki et al., 1988).

Exact neuronal mechanisms for the sensitivity of the pyramidal cells to these extraordinarily small time disparities are unknown. The following observations and theoretical considerations have been made in an attempt to understand the sensitivity. The SD of firing times of phase-locking neurons that project to the ICL is $5.6 \mu$ s on average, and $95 \%$ of them show SDs $<10$ $\mu \mathrm{s}$ (Guo and Kawasaki, 1997). At their thresholds, pyramidal cells required several seconds of continuous stimulus presentation, during which thousands of accurately phase-locked action potentials arrive at the time comparator (Fig. 8). Statistically speaking, the sample $\mathrm{SD}$ is $1 / \sqrt{n}$, where $n$ is the number of action potentials given by a phase-locking neuron to the comparator during the several seconds. If a pyramidal cell receives action potentials at $400 \mathrm{~Hz}$ for $2 \mathrm{~s}$, for example, the sample SD would be $0.2 \mu \mathrm{s}(5.6 / \sqrt{800})$. This means that rather precise time information would be available to comparator neurons. Specialized anatomical structures have been found in the ICL. Terminals of the giant cells form a giant synapse that embraces $\sim 85 \%$ of the soma area of the postsynaptic neurons, termed ovoidal cells (Matsushita and Kawasaki, 2004). This morphology may enhance the rapidity of synaptic transmission due to ephaptic effects (Byzov and Shura-Bura, 1986; Voronin et al., 1999). No matter how accurate the firing times of input phase-locking neurons are, synaptic currents produced by these inputs are expected to have much longer time constants than the threshold sensitivity of pyramidal cells to time disparities (Trussell, 1999). Takagi and Kawasaki (2003) showed that the Hodgkin-Huxley equation may yield sensitivity to time disparity on the order of $10^{-5} \mathrm{~s}$ between two synaptic input currents with a much larger time constant $(\sim 300 \mu \mathrm{s})$ due to the nonlinear dynamics of the equation.

Neuronal representation and processing of temporal information on the order of microseconds has been found not only in the electrosensory system (Heiligenberg and Altes, 1978; Rose and Heiligenberg, 1986; Kawasaki et al., 1988) but also in the auditory system of birds and mammals. Phase-locking neurons in the nucleus magnocellularis of the barn owl (Carr and Konishi, 1990), and those in the medial superior olive and the trapezoid body of mammals (Joris et al., 1994), show only a few tens of microseconds of jitter. Although statistical examinations have not been made for threshold determination, auditory neurons in the midbrain of birds and mammals appear to show considerable sensitivities to interaural time disparities of tens of microseconds (Goldberg and Brown, 1969; Yin and Kuwada, 1983; Takahashi and Konishi, 1986; Yin et al., 1986, 1987; Chan et al., 1987). Skottun et al. (2001) and Shackleton et al. (2003) applied a statistical method, the receiver operating characteristic analysis, to interaural time-disparity-sensitive neurons in guinea pig and demonstrated thresholds around $30 \mu \mathrm{s}$. The time-disparity thresholds demonstrated in the pyramidal cells of Gymnarchus in the current study are $\sim 5-10$ times lower than those found in the auditory midbrain of birds and mammals.

We regard the units we recorded from as pyramidal cells in the ICL or OCL for the following reasons. First, the latencies of their action potentials from the peak of the phase-locking field potential were consistent with previous results using in vivo whole-cell recording and labeling, which confirmed the morphology of the 


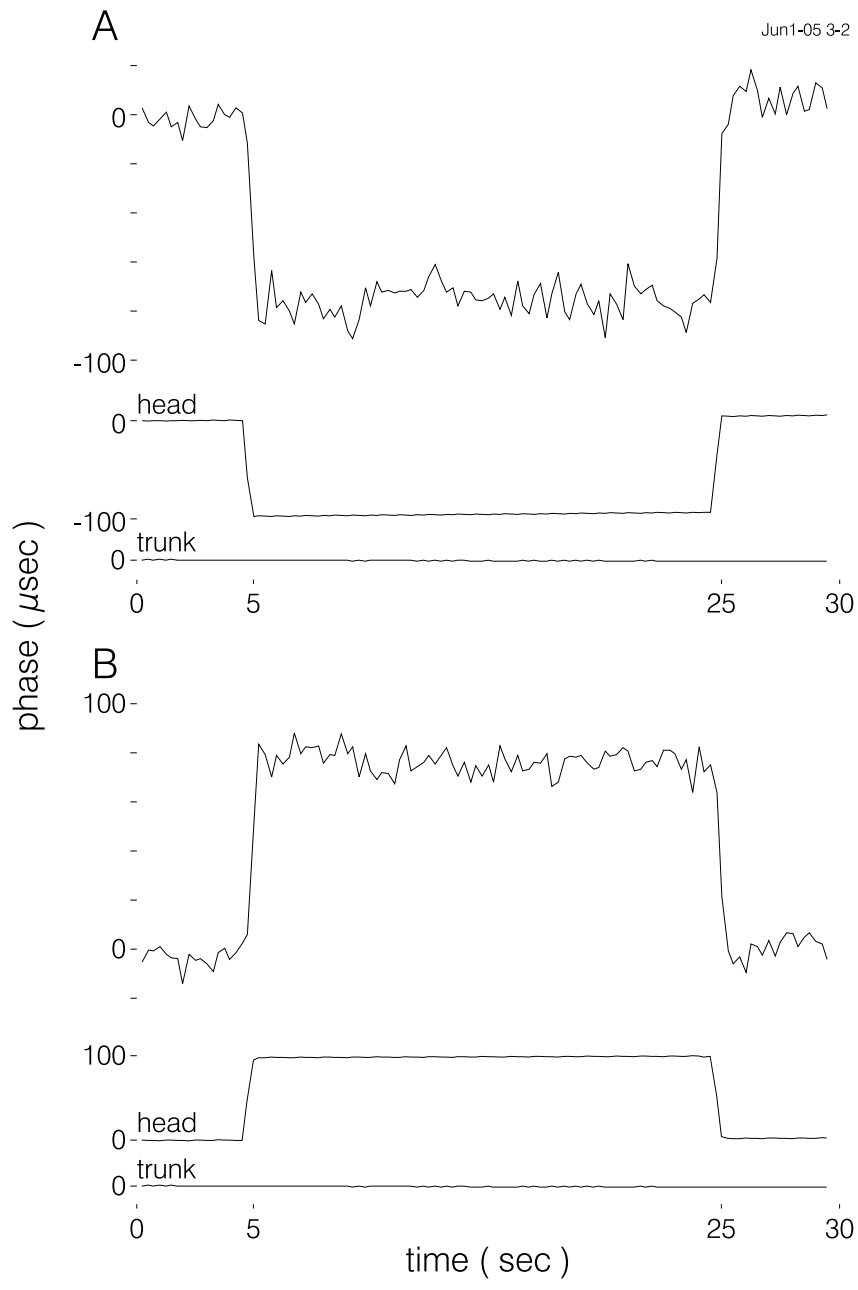

Figure 10. Changes in spike times of a phase-locking neuron in the DFL (top trace) in response to steady phase shifts of the carrier signal (bottom traces). After an initial $5 \mathrm{~s}$, the phase of the carrier signal to the head compartment was shifted to $-100 \mu \mathrm{s}(\boldsymbol{A})$ or $+100 \mu \mathrm{s}(\boldsymbol{B})$ and held there for 20 s and then moved back to zero. The time for shifting was set at $0.5 \mathrm{~s}$. In response to the phase shift, the spike times shifted and remained there during the $20 \mathrm{~s}$ period, showing no adaptation. The smaller magnitude of shifts in neuronal response compared with the stimulus is likely because only a part of the large receptive field of the neuron was exposed to the phase-shifted stimulus in the phase chamber.

recorded cell as pyramidal cells (Kawasaki and Guo, 1996, 1998), whereas the latency of the ovoidal cell is expected to be shorter than that of pyramidal cells. Second, the recording sites were shallower than the ovoidal cell layer, based on the waveform of the concurrently recorded field potential (Fig. 3 ) as well as actual depth in the ELL (Kawasaki and Guo, 1996). Third, the large action potentials are likely to be generated by the large apical dendrites of pyramidal cells (Bell et al., 1993; Kawasaki and Guo, 1998), which the ovoidal cells do not possess (Matsushita and Kawasaki, 2004).

It is remarkable that there was no significant difference in sensitivity to time disparity between ICL and OCL pyramidal cells given their different anatomical position: the pyramidal cells in the ICL receive phase-locked inputs, whereas those in the OCL do not. This anatomical difference reflects the difference in their vector strength, $V s$, and firing latency. A possible explanation for the similarity of sensitivity is that the output of ovoidal cells may be given to pyramidal cells in the ICL via their dendrite and those in the OCL via their axon (Fig. 1) (Matsushita and Kawasaki, 2004).

\section{Adaptation property of pyramidal cells}

The pyramidal cells preserved their sensitivity to near-threshold DPM $(5 \mu \mathrm{s})$ over a wide range of center phase (current study) as well as suprathreshold DPM (Kawasaki and Guo, 1996). The arrival times of action potentials of phase-locking neurons to the pyramidal cells depends on the conduction velocity of the neuronal path between the electroreceptors and pyramidal cells, which may easily vary in natural conditions because of temperature changes. Local temperature changes may occur if a fish stayed at a boundary between sun and shade (such as vegetation), resulting in a change of center phase experienced by a phase comparator. One degree difference of body temperature between head and trunk would result in a few hundred microseconds of conduction time difference along $10 \mathrm{~cm}$ of the body length of a fish (Nagy et al., 1978). The range of $250 \mu$ s of center phase in which the neuron retains sensitivity to threshold DPM5 is similar to the value in Eigenmannia (Rose and Heiligenberg, 1986) and may be reasonable to compensate such delays. Behavioral study in Eigenmannia showed that correct JAR occurred in response to a combination of small phase modulation $(5 \mu \mathrm{s})$ and amplitude modulation $(0.1 \%)$ with center phase shifted by $>100 \mu$ s (our unpublished observation).

The phase-locking neurons in the DFL we recorded in this study were either S-afferents or giant cells. These neurons responded to the dynamic shifts of phase but did not show any adaptation over a period of $20 \mathrm{~s}$, which is enough time for pyramidal cells to adapt (Kawasaki and Guo, 1996). The giant cell terminal gives a synaptic input to ovoidal cells, whereas S-afferents make synaptic contacts directly onto pyramidal cells in the ICL and ovoidal cells (Matsushita and Kawasaki, 2004). Thus, the adaptation may emerge at the level of pyramidal cells or ovoidal cells. The morphology of the pyramidal cells in both the ICL and OCL is similar to the Purkinje-like cells in mormyrid electric fish (Grant et al., 1996), whose large apical dendrites receive descending inputs from higher centers to cancel out predictable sensory inputs to the cell (Bell et al., 1993). Similar mechanisms for adaptively canceling steady-state phase shifts might be involved in Gymnarchus.

\section{References}

Bass AH, Hopkins CD (1982) Comparative aspects of brain organization of an African "wave" electric fish, Gymnarchus niloticus. J Morphol 174:313-334.

Bastian J, Heiligenberg W (1980) Phase-sensitive midbrain neurons in Eigenmannia: neural correlates of the jamming avoidance response. Science 209:828-831.

Batschelet E (1981) Circular statistics in biology, pp 55-61. New York: Academic.

Bell CC, Caputi A, Grant K, Serrier J (1993) Storage of a sensory pattern by anti-Hebbian synaptic plasticity in an electric fish. Proc Natl Acad Sci USA 90:4650-4654.

Bullock TH, Hamstra RH, Scheich H (1972) The jamming avoidance response of high frequency electric fish. I. General features. J Comp Physiol 77:1-22.

Bullock TH, Behrend K, Heiligenberg W (1975) Comparison of the jamming avoidance responses in Gymnotoid and Gymnarchid electric fish: a case of convergent evolution of behavior and its sensory basis. J Comp Physiol 103:97-121.

Byzov AL, Shura-Bura TM (1986) Electrical feedback mechanism in the processing of signals in the outer plexiform layer of the retina. Vision Res 26:33-44

Carr CE, Friedman MA (1999) Evolution of time coding systems. Neural Comput 11:1-20.

Carr CE, Konishi M (1990) A circuit for detection of interaural time differences in the brain stem of the barn owl. J Neurosci 10:3227-3246.

Carr CE, Soares D, Parameshwaran S, Perney T (2001) Evolution and development of time coding systems. Curr Opin Neurobiol 11:727-733. 
Chan JC, Yin TC, Musicant AD (1987) Effects of interaural time delays of noise stimuli on low-frequency cells in the cat's inferior colliculus. II. Responses to band-pass filtered noises. J Neurophysiol 58:543-561.

Dowben R, Rose J (1953) A metal-filled microelectrode. Science 118:22-24.

Fu J, Lorden JF (1996) An easily constructed carbon fiber recording and microiontophoresis assembly. J Neurosci Methods 68:247-251.

Goldberg JM, Brown PB (1969) Response of binaural neurons of dog superior olivary complex to dichotic tonal stimuli: some physiological mechanisms of sound localization. J Neurophysiol 32:613-636.

Grant K, Meek J, Sugawara Y, Veron M, Denizot JP, Hafmans TG, Serrier J, Szabo T (1996) Projection neurons of the mormyrid electrosensory lateral line lobe: morphology, immunohistochemistry, and synaptology. J Comp Neurol 375:18-42.

Guo Y-X, Kawasaki M (1997) Representation of accurate temporal information in the electrosensory system of the African electric fish, Gymnarchus niloticus. J Neurosci 17:1761-1768.

Heiligenberg W, Altes RA (1978) Phase sensitivity in electroreception. Science 199:1001-1004.

Heiligenberg W, Bastian J (1980) The control of Eigenmannia's pacemaker by distributed evaluation of electroreceptive afferences. J Comp Physiol 136:113-133.

Joris PX, Smith PH, Yin TC (1994) Enhancement of neural synchronization in the anteroventral cochlear nucleus. II. Responses in the tuning curve tail. J Neurophysiol 71:1037-1051.

Kawasaki M (1993) Independently evolved jamming avoidance responses employ identical computational algorithms: a behavioral study of the African electric fish, Gymnarchus niloticus. J Comp Physiol A Neuroethol Sens Neural Behav Physiol 173:9-22.

Kawasaki M (1994) The African wave-type electric fish, Gymnarchus niloticus, lacks corollary discharge mechanisms for electrosensory gating. J Comp Physiol A Neuroethol Sens Neural Behav Physiol 174:133-144.

Kawasaki M, Guo Y-X (1996) Neuronal circuitry for comparison of timing in the electrosensory lateral line lobe of the African wave-type electric fish Gymnarchus niloticus. J Neurosci 16:380-391.

Kawasaki M, Guo Y-X (1998) Parallel projection of amplitude and phase information from the hindbrain to the midbrain of the African electric fish Gymnarchus niloticus. J Neurosci 18:7599-7611.

Kawasaki M, Guo Y-X (2002) Emergence of temporal-pattern sensitive neurons in the midbrain of weakly electric fish Gymnarchus niloticus. J Physiol (Paris) 96:531-537.

Kawasaki M, Rose GJ, Heiligenberg W (1988) Temporal hyperacuity in single neurons of electric fish. Nature 336:173-176.

Knudsen EI, Blasdel GG, Konishi M (1979) Sound localization by the barn owl (Tyto alba). J Comp Physiol 133:13-21.

Lauder GV, Liem KF (1983) Patterns of diversity and evolution in rayfinned fishes. In: Fish neurobiology (Northcutt RG, Davis RE, eds), pp 1-24. Ann Arbor, MI: University of Michigan.

Mason AC, Oshinsky ML, Hoy RR (2001) Hyperacute directional hearing in a microscale auditory system. Nature 410:686-690.
Matsushita A, Kawasaki M (2004) Unitary giant synapses embracing a single neuron at the convergent site of time-coding pathways of an electric fish, Gymnarchus niloticus. J Comp Neurol 472:140-155.

Nagy L, Lakatos T, Tegzes L (1978) Temperature dependence of conduction velocity in myelinated frog nerve. Acta Biochim Biophys Acad Sci Hung 13:299-306.

Rose GJ, Heiligenberg W (1985) Temporal hyperacuity in the electric sense of fish. Nature 318:178-180.

Rose GJ, Heiligenberg W (1986) Limits of phase and amplitude sensitivity in the torus semicircularis of Eigenmannia. J Comp Physiol A Neuroethol Sens Neural Behav Physiol 159:813-822.

Rose GJ, Kawasaki M, Heiligenberg W (1988) 'Recognition units' at the top of a neuronal hierarchy? Prepacemaker neurons in Eigenmannia code the sign of frequency differences unambiguously. J Comp Physiol A Neuroethol Sens Neural Behav Physiol 162:759-772.

Shackleton TM, Skottun BC, Arnott RH, Palmer AR (2003) Interaural time difference discrimination thresholds for single neurons in the inferior colliculus of guinea pigs. J Neurosci 23:716-724.

Simmons JA, Ferragamo MJ, Sanderson MI (2003) Echo delay versus spectral cues for temporal hyperacuity in the big brown bat, Eptesicus fuscus. J Comp Physiol A Neuroethol Sens Neural Behav Physiol 189:693-702.

Skottun BC, Shackleton TM, Arnott RH, Palmer AR (2001) The ability of inferior colliculus neurons to signal differences in interaural delay. Proc Natl Acad Sci USA 98:14050-14054.

Takagi H, Kawasaki M (2003) Modeling of time disparity detection by the Hodgkin-Huxley equations. J Comp Physiol A Neuroethol Sens Neural Behav Physiol 189:257-262.

Takahashi T, Konishi M (1986) Selectivity for interaural time difference in the owl's midbrain. J Neurosci 6:3413-3422.

Theunissen F, Miller JP (1995) Temporal encoding in nervous systems: a rigorous definition. J Comput Neurosci 2:149-162.

Trussell LO (1999) Synaptic mechanisms for coding timing in auditory neurons. Annu Rev Physiol 61:477-496.

Voronin LL, Volgushev M, Sokolov M, Kasyanov A, Chistiakova M, Reymann KG (1999) Evidence for an ephaptic feedback in cortical synapses: postsynaptic hyperpolarization alters the number of response failures and quantal content. Neuroscience 92:399-405.

Watanabe A, Takeda K (1963) The change of discharge frequency by AC stimulus in a weakly electric fish. J Exp Biol 40:57-66.

Yin TC, Kuwada S (1983) Binaural interaction in low-frequency neurons in inferior colliculus of the cat. III. Effects of changing frequency. J Neurophysiol 50:1020-1042.

Yin TC, Chan JC, Irvine DR (1986) Effects of interaural time delays of noise stimuli on low-frequency cells in the cat's inferior colliculus. I. Responses to wideband noise. J Neurophysiol 55:280-300.

Yin TC, Chan JC, Carney LH (1987) Effects of interaural time delays of noise stimuli on low-frequency cells in the cat's inferior colliculus. III. Evidence for cross-correlation. J Neurophysiol 58:562-583. 